OPEN ACCESS

Edited by:

George Grant,

University of Aberdeen,

United Kingdom

Reviewed by:

Subash C. Sonkar

University of Delhi, India

Robert V. Schoborg,

East Tennessee State University,

United States

Virginia Elena Rivero,

CONICET Centro de Investigaciones en Bioquímica Clínica e Inmunología

(CIBICl), Argentina

${ }^{*}$ Correspondence:

Shixing Tang

tamgshixing@smu.edu.cn

Zhongyu L

Izhy1023@hotmail.com

Specialty section:

This article was submitted to

Infectious Diseases,

a section of the journal

Frontiers in Microbiology

Received: 29 January 2020 Accepted: 07 April 2020

Published: 05 May 2020

Citation:

Chen H, Luo L, Wen Y, He B,

Ling $H$, Shui J, He P, Hou $X$, Tang $S$ and Li Z (2020) Chlamydia

trachomatis and Human

Papillomavirus Infection in Women From Southern Hunan Province in China: A Large Observational Study. Front. Microbiol. 11:827. doi: 10.3389/fmicb.2020.00827

\section{Chlamydia trachomatis and Human Papillomavirus Infection in Women From Southern Hunan Province in China: A Large Observational Study}

\author{
Hongliang Chen 1,2, Lipei Luo 2,4, Yating Wen', Bei He', Hua Ling2,4, Jinwei Shui ${ }^{5}$, \\ Ping $\mathrm{He}^{2}$, Xiaoli Hou${ }^{2}$, Shixing Tang ${ }^{3,5 *}$ and Zhongyu $\mathrm{Li}^{1 *}$
}

\begin{abstract}
1 Institute of Pathogenic Biology, Hengyang Medical College, Hunan Provincial Key Laboratory for Special Pathogens Prevention and Control, Hunan Province Cooperative Innovation Center for Molecular Target New Drug Study, University of South China, Hengyang, China, ${ }^{2}$ Department of Clinical Microbiology Laboratory, Chenzhou No. 1 People's Hospital, Chenzhou, China, ${ }^{3}$ Dermatology Hospital, Southern Medical University, Guangzhou, China, ${ }^{4}$ Affiliated Chenzhou Hospital, Southern Medical University, Guangzhou, China, ${ }^{5}$ Department of Epidemiology, School of Public Health, Southern Medical University, Guangzhou, China
\end{abstract}

Chlamydia trachomatis and human papillomavirus (HPV) are the most common pathogens of sexually transmitted infections (STIS), which can increase the risk of cervical cancer and infertility. The purpose of this study was to evaluate the prevalence, genotype and risk factors of $C$. trachomatis and/or HPV infection in women attending the annual physical examination, assistant reproductive treatment and visiting the gynecology clinics from Southern Hunan province in China. Cervical-swab samples were collected from 5006 participants. We found that the overall prevalence of $C$. trachomatis, HPV infection and C. trachomatis/HPV coinfection was 4.7\% (236/5006), 15.5\% (778/5006) and $1.2 \%$ (59/5006), while the prevalence of asymptomatic infection of that was 3.8\% (38/1006), 10.8\% (109/1006) and 0.6\% (6/1006), respectively. Furthermore, $25.0 \%(59 / 236)$ of C. trachomatis infection and $7.6 \%(59 / 778)$ of HPV infection were attributable to $C$. trachomatis and HPV coinfection. C. trachomatis and HPV infection were more often observed in young women of less than 25 years (10.4\% and $21.3 \%$, respectively) and in the outpatients from gynecology clinics (5.2\% and $18.0 \%$, respectively). Of note, a higher prevalence of $C$. trachomatis infection was observed in HPV-positive women (7.6\%) than HPV- negative ones (4.2\%), and vice versa. The top three $C$. trachomatis genotypes were $E(1.4 \%), F(1.1 \%)$ and $\mathrm{J}(0.8 \%)$, and the counterparts of HPV genotypes were HPV52 (4.2\%), HPV16 (2.3\%) and HPV58 (2.2\%), respectively. Among the 151 outpatients with colposcopy data, HPV infection was associated with severe cervical lesions with OR of 15.86 (95\% Cl 3.14-80.0, $P<0.001$ ) while $C$. trachomatis infection was more likely associated with a low grade colposcopy impression (OR $=3.25,95 \% \mathrm{Cl}: 1.22-8.65, P=0.018)$. Our data highlight the high prevalence of asymptomatic $C$. trachomatis and HPV infection, particularly 
among women of $<25$ years. The two pathogens may serve as mutual risk factors to increase the risk of infections and cervical lesions. Widespread implementation of HPV and C. trachomatis screening programs, especially for young women, would be an effective strategy to relieve the burden of sexually transmitted infections.

Keywords: Chlamydia trachomatis, human papillomavirus, coinfection, prevalence, genotype distribution

\section{INTRODUCTION}

Chlamydia trachomatis (CT), an obligate intracellular parasitic bacterium, is the leading bacterial cause of sexually transmitted infections (STIs) (Witkin et al., 2017) while human papillomavirus (HPV) has been documented to cause cervical cancer (Harden and Munger, 2017). It is estimated that 101 million new cases of $C$. trachomatis occur annually worldwide, causing important health problems. Actually, the number of C. trachomatis-infected population may be more than the reported cases since $80 \%$ of $C$. trachomatis infection may be asymptomatic and show subclinical course. This microorganism can be subdivided into at least 19 serotypes (Rawre et al., 2017; Hadfield et al., 2018). Serovars A to C are mainly related to trachoma; serovars D-K usually cause urogenital infections (Geisler et al., 2003), such as urethritis, cervicitis, pelvic inflammatory disease, and ectopic pregnancy; and serovars L1-L3 are associated with lymphogranuloma venereum.

Epidemiological studies have shown that C. trachomatis infection has also been identified as an independent risk factor of cervical cancer, indicating a potential relationship with HPV infection (Bellaminutti et al., 2014; Di Pietro et al., 2018). In terms of pathogenesis, C. trachomatis is capable of triggering chronic or recurrent infections and long-term inflammations of the urethra (Chen et al., 2019), inducing local secretion of immune mediators, enhancing the production of reactive oxygen species (ROS) and generation of free radicals, which may cause damages to host mucosal barriers and cell-mediated immunity (CondeFerraez et al., 2017). These biological effects of C. trachomatis infection could facilitate the transmission and co-infection of HPV and decrease the host's ability to resolve HPV infection.

$\mathrm{HPV}$ is the most common viral infection of the reproductive tract and is also a well-established risk factor for cervical cancer (Schiffman et al., 2016; Clarke et al., 2018). To date, over $100 \mathrm{HPV}$ genotypes have been identified, and are associated with cervical lesions. Infections with low-risk HPV (LrHPV) genotype, such as HPV types 6 and 11, can cause benign warts and recurrent respiratory papillomatosis. High-risk HPV (HrHPV) types are closely associated with cervical, anal, and other genital cancers (Harden and Munger, 2017). The overall HrHPV infection rate in China is over $89 \%$ in patients with cervical cancer, ranging from $81 \%$ in North to $94 \%$ in Southwest of China (Li et al., 2019).

Both HPV and C. trachomatis are sexually transmitted, sharing common transmission routes and the same risk factors. Several studies have found that $C$. trachomatis promotes HPV persistence, and is associated with cervical cancer (Bhatla et al., 2013). However, the effects and clinical consequences of C. trachomatis and HPV coinfection are poorly explored. Understanding the prevalence, genotype distribution and risk factors of C. trachomatis and HPV infection, especially for asymptomatic infection, are critical for effective prevention and interventions.

Since C. trachomatis has not yet been included as a reportable STI in the national STI surveillance program in China, the detailed epidemic data is lacking. The first nationwide population-based C. trachomatis infection investigation in 19992000 found a prevalence of $2.1 \%$ among men and $2.6 \%$ among women (Parish et al., 2003), whereas women with secondary infertility have a relatively high prevalence of $13.3 \%$ (Shi et al., 2001). Furthermore, the HPV prevalence varies significantly and ranges from 16.8-36\% in China (Li et al., 2006; Ji et al., 2019). Considering the geographic variation of C. trachomatis and HPV infection, we thus enrolled a large number of women from Southern Hunan province in China, including healthy women attending the annual physical examination, or young women seeking reproductive assistance and outpatients in the gynecology clinics to investigate prevalence and risk factors of C. trachomatis and HPV infection. Such data may provide insights into the epidemiology of C. trachomatis and HPV infection in the region.

\section{MATERIALS AND METHODS}

\section{Participants and Clinical Samples}

During December 2018 and November 2019, cervical-swab samples were collected for detecting C. trachomatis and HPV from three groups of Chinese women, i.e., the seemingly healthy women to receive annual physical examination in the physical examination center (PEC); women seeking for assistance at the assisted reproductive technology center (ART); and outpatients from the gynecology clinics (GC), respectively. The inclusion criteria are female, not taking antibiotics, not being pregnant, and no parturition when sampling. Asymptomatic C. trachomatis/HPV infection was defined as positive for $C$. trachomatis/HPV nucleic acid detection but without symptoms, such as painful sexual intercourse, abnormal vaginal discharge, urethritis, irregular vaginal bleeding, or bleeding after sexual intercourse and genital warts (Wiesenfeld et al., 2005; Erickson et al., 2013). Asymptomatic participants were mainly from ART. Cervicalswab samples were collected using a $200 \mathrm{~mm}$ polyethylene Cervix brush device (Hybribio Corp, Guangdong) following the regular procedures for speculum examination. The specimens were transferred to a tube containing cervical cell preservation solution provided in the kit and stored at room temperature for immediate processing or $-80^{\circ} \mathrm{C}$ until analysis. Cervical samples can be kept in the transport medium for 2 weeks at $4^{\circ} \mathrm{C}$ according to the manufacturer's manual. 
The study was conducted in the teaching hospital, Chenzhou No.1 People's Hospital, in Chenzhou, and was under the Principles of the Declaration of Helsinki. It was approved by The Ethics Committee of Chenzhou No.1 People's Hospital (CZ/1128). Written inform consents were obtained from the participants. All experiments were carried out in the lab certified by the National Center for Clinical Laboratories following the laboratory biosafety guidelines.

\section{DNA Extraction}

DNA was extracted from cervical-swabs samples within $48 \mathrm{~h}$ after collection using the QIAamp mini kit (Qiagen). DNA isolation and purification were conducted according to the manufacturer's instructions. Briefly, the samples were centrifuged at $14000 \mathrm{rpm}$ for $1 \mathrm{~min}$. The supernatant was removed and $20 \mu \mathrm{L}$ protein $\mathrm{K}$ was added, following by incubation for $10 \mathrm{~min}$ at $37^{\circ} \mathrm{C}$. DNA was immediately amplified by polymerase chain reaction (PCR) or stored at $-20^{\circ} \mathrm{C}$ for further analysis. The concentration and purity of the extracted DNA were determined by using Thermo Scientific Varioskan Flash Spectral Scanning Multimode Reader (Thermo Fisher Scientific, Inc.). Water control was processed in parallel to monitor contamination during DNA extraction. The human housekeeping gene $\beta$-globin was used as an endogenous internal control to ensure the quality of DNA and the efficiency of PCR.

\section{HPV Genotyping}

HPV detection and genotyping were performed by using the Hybribio Rapid Geno-Array test kit (Hybribio Corp, Guangdong) based on the PCR-reverse dot blot hybridization method. The low limit of detection (LOD) for HPV DNA is 500 copies/ml according to manufacturer's protocol. The kit detects $21 \mathrm{HPV}$ genotypes, including $15 \mathrm{HrHPV}$ genotypes (types 16, $18,31,33,35,39,45,51,52,53,56,58,59$, and 68), and 6 LrHPV genotypes (types 6, 11, 42, 43, 44, and CP8304 [81]). This assay uses HPV L1 consensus PCR primers (MY09/11) for the amplification. HPV genotypes were then determined through hybridization using genotype-specific probes (Ji et al., 2019).

\section{C. trachomatis Detection and Genotyping}

A 200 bp conserved cryptic plasmid fragment of C. trachomatis DNA was amplified for diagnosis of $C$. trachomatis infection with the following primes: CT1: $5^{\prime}$-TTCCCCTTGTAATTCGTTGC$3^{\prime}$ and CT2: 5'-TAGTAACTGCCACTTCATCA-3'. PCR was carried out in $25 \mu \mathrm{l}$ reaction mixture in a thermal cycler, the reaction conditions for which were $95^{\circ} \mathrm{C}$ for $5 \mathrm{~m}$, followed by 35 cycles of $95^{\circ} \mathrm{C}$ for $50 \mathrm{~s}, 55^{\circ} \mathrm{C}$ for $45 \mathrm{~s}$ and $72^{\circ} \mathrm{C}$ for $45 \mathrm{~s}$, with a final elongation at $72^{\circ} \mathrm{C}$ for $10 \mathrm{~min}$ as previously described (Griffais and Thibon, 1989). The cryptic plasmid-based PCR showed a LOD of 400 copies $/ \mathrm{ml}$ and a concordance rate of $95 \%$ with a commercial C. trachomatis molecular diagnostic kit (Sansure Biotech, China).

For C. trachomatis genotyping, an approximate $1100 \mathrm{bp}$ fragment encompassing VS1-VS4 of C. trachomatis omp1 gene was amplified using nested PCR with the following primers: outer primes omp1 P1: 5'-CTCAACTGTAACTGCGTATTT-3' and omp1 P2: 5'-ATGAAAAAACTCTTGAAATCG-3'. PCR was carried out in $25 \mu \mathrm{l}$ reaction mixture with the following reaction conditions: $95^{\circ} \mathrm{C}$ for $5 \mathrm{~m}$, followed by 25 cycles of $95^{\circ} \mathrm{C}$ for $60 \mathrm{~s}, 55^{\circ} \mathrm{C}$ for $60 \mathrm{~s}$ and $72^{\circ} \mathrm{C}$ for $80 \mathrm{~s}$, with a final elongation at $72^{\circ} \mathrm{C}$ for $10 \mathrm{~min}$. An approximate $580 \mathrm{bp} \mathrm{VS1-VS2} \mathrm{fragment}$ was further amplified using the second-round PCR with the inner primer sets P3: 5' - TGAACCAAGCCTTATGATCGACGGA-3' and omp1 P2: 5' -TCTTCGAYTTTAGGTTTAGATTGA-3', and the reaction conditions: $95^{\circ} \mathrm{C}$ for $5 \mathrm{~m}$, followed by 35 cycles of $95^{\circ} \mathrm{C}$ for $30 \mathrm{~s}, 55^{\circ} \mathrm{C}$ for $30 \mathrm{~s}$ and $72^{\circ} \mathrm{C}$ for $30 \mathrm{~s}$, with a final elongation at $72^{\circ} \mathrm{C}$ for $10 \mathrm{~min}$ ( $\mathrm{Hsu}$ et al., 2006). The C. trachomatis (ATCC VR-348B) was used as a positive control and DNase-free water as a negative control in PCR. Positive PCR products for C. trachomatis omp1 VS1-VS2 were sequenced bidirectionally in Ruibo, Beijing. Genotypes of C. trachomatis were determined using BLAST program ${ }^{1}$ as previously described (Hsu et al., 2006; Yang et al., 2010).

\section{Colposcopy Test}

Colposcopy screening was performed using a digital electronic colposcope (SLC-3000, Philips, Shenzhen) following a standard procedure. According to the standard and terminology of the American Society for Colposcopy and Cervical Pathology (ASCCP) (Khan et al., 2017), colposcopy impression includes benign, low grade features, high grade features, and cancer. Lowgrade and high-grade features correspond to Grade 1 (minor) and Grade 2 (major) abnormal colposcopy findings defined by the International Federation for Cervical Pathology and Colposcopy (IFCPC) nomenclature.

\section{Statistical Analysis}

Statistical analyses were conducted using SPSS version 19.0 software. Chi-Square Test was used to compare frequencies of discrete variables. The Fisher Exact Test was applied when necessary. Bivariate logistic regression was used to assess the risk factors affecting the prevalence of $C$. trachomatis and HPV. Statistical differences were considered being significant when $P<0.05$.

\section{RESULTS}

\section{Prevalence of C. trachomatis and HPV Infection}

From December 2018 to November 2019, a total of 5027 women were enrolled in our study, and 21 participants were excluded due to poor sample quality or lack of personal information. Therefore, 5006 women with cervical swab samples were analyzed. Among them, 1006 women were from PEC, 666 from ART and 3334 outpatients from GC (Table 1). The overall prevalence of $C$. trachomatis and HPV infection was 4.7\% $(236 / 5006)$ and $15.5 \%$ (778/5006), respectively. The prevalence of C. trachomatis infection was higher in the outpatients $(5.2 \%)$ from GC than in those from PEC (3.8\%) or ART (3.5\%)

\footnotetext{
${ }^{1}$ http://www.ncbi.nlm.nih.gov/BLAST
} 
$(P=0.043$, Table 1$)$, and was also higher in the group of $\leq 25$ years than in those of $>25$ years ( 10.4 vs $4.0 \%, P<0.001$, Table 1 and Supplementary Table S1). The age-related difference of C. trachomatis infection was supported by multivariate analysis (Table 2) and existed among the participants from different departments (Supplementary Table S1). These results indicated that young women of less than 25 years and the patients in the gynecology clinic are the risk group for C. trachomatis infection.

Similar results were obtained for HPV infection, which was more often detected in the outpatients $(18.0 \%)$ from GC than in those from PEC $(10.8 \%)$ or ART $(10.4 \%)(P<0.001$, Table 1$)$, and in the group of $\leq 25$ years than in those of $>25$ years (21.3 vs $14.8 \%, P<0.001$, Supplementary Table S1). However, the prevalence of HPV infection was not significantly different between the age group of $>25$ years and $\leq 25$ years for those seeking annual physical examination $(P=0.156)$ and assistant reproduction treatment $(P=0.565$, Supplementary Table S1).

\section{Co-infection of C. trachomatis and HPV}

C. trachomatis and HPV coinfection was only detected in $1.2 \%(59 / 5006)$ of the participants and more often observed in women aged $\leq 25$ years than in those of $>25$ years (3.8 vs $0.8 \%, P<0.001)$. However, the prevalence of $C$. trachomatis and HPV co-infection was not significantly different among the women from PEC (0.6\%), ARC (0.8\%) and GC (1.4\%) $(P=0.053)$ (Table 1).

Among the 236 participants infected with C. trachomatis, 59 (25.0\%) were co-infected with HPV. We also noted a higher prevalence of $C$. trachomatis in HPV positive women $(7.6 \%$, 59/778) than HPV negative ones $(4.2 \%, 177 / 4235)$ with an odds ratio of 1.72 (95\% CI: 1.254-2.332, $P<0.001$ ) (Table 2 and Supplementary Table S4). For the 778 participants infected with HPV, 59 (7.6\%) were co-infected with C. trachomatis. The prevalence of HPV infection was $25.0 \%$ (59/236) in C. trachomatis positive women and $15.2 \%(719 / 4770)$ in C. trachomatis negative women with an odds ratio of $1.72(95 \%$ CI: 1.259-2.339, $P<0.001$ ) (Table 2 and Supplementary Table S5). These results indicate the tight association of C. trachomatis and HPV infection. Such an association was further confirmed by a case-control study in which $C$. trachomatis positive cases and controls were matched by age and population groups. We found that $C$. trachomatis infection was associated with high risk of HPV infection with an odds ratio of 1.74 (95\% CI 1.10-2.74, $P=0.017$ ). Similar results were observed for the enhanced $C$. trachomatis infection among HPV-infected women with an odds ratio of 1.69 (95\% CI 1.10-2.59, $P=0.015)$ (Supplementary Table S4 and S6).

\section{Genotyping of C. trachomatis and HPV}

The VS1-S2 fragment of $C$. trachomatis Omp1 gene was successfully amplified from 236 C. trachomatis-positive samples and 229 discernible sequences were classified into 8 genotypes. The top three C. trachomatis genotypes were E $(1.4 \%, 69 / 5006)$, F $(1.1 \%, 53 / 5006)$ and $J(0.8 \%, 41 / 5006)$, accounting for 30.1 , 23.1, and $17.9 \%$ of C. trachomatis-positive samples (Table 3). No significant difference was observed in the distribution of C. trachomatis genotypes among the participants from different clinical departments $(P=0.78$, Table 3$)$. Interestingly, one sample was originally classified as subtype B of C. trachomatis according to the Omp1 gene and was further confirmed by multilocus sequence typing (MLST) to be infected with subtype B (data not shown).

Furthermore, the overall prevalence of different HPV genotype infection was as follows: $12.9 \%$ (648/5006) for HrHPVonly, $1.5 \%(77 / 5006)$ for LrHPV-only and $1.1 \%(53 / 5006)$ for the superinfection of HrHPV and LrHPV, respectively (Table 4). As shown in Figure 1, the most common HrHPV genotype was HPV52 (4.2\%), followed by HPV16 (2.3\%), HPV58 (2.2\%), HPV39 (1.6\%), HPV51 (1.6\%), HPV53 (1.4\%), HPV33 (0.8\%), HPV18 (0.7\%), HPV68(0.6\%), HPV31 (0.6\%), HPV 59 (0.4\%), HPV56 (0.4\%), HPV66 (0.3\%), HPV45 (0.2\%) and HPV35 (0.1\%). For the LrHPV genotypes, the most common type was HPV83 (1.1\%), followed by HPV6 (1.0\%), HPV 11 (0.4\%), HPV44 (0.3\%), HPV43 (0.2\%) and HPV42 $(0.1 \%)$. There was no significant difference of both HrHPV and LrHPV genotypes distribution among different clinical departments $(P=0.45)$, various age groups $(P=0.61)$ and between $C$. trachomatis positive or negative women $(P=0.24$, Table 4). However, HrHPV only infection was more often detected in C. trachomatis positive women (22.0\%), outpatients in GC (14.6\%) and young females of $<25$ years $(16.2 \%)$ (Table 4), suggesting that these women may be more prone to HrHPV infection.

Also, 77.4\% (602/778) of HPV positive participants infected with only one HPV genotype while $17.4 \%$ (136/778) and $4.6 \%$ (36/778) of them infected with 2 and 3 HPV genotypes, respectively. There were only 5 cases $(0.6 \%)$ infected with more than $4 \mathrm{HPV}$ genotypes (Supplementary Table S2). We noted a significant difference in the clinical departmentspecific distribution of single and duplicate genotype infections $(P<0.01$ and $P<0.05)$. HPV52 $(137,17.6 \%)$ accounted for the most common HrHPV genotypes in those with a single genotype of HPV infection, followed by HPV16 (74, 9.5\%), and HPV58 (68, 8.6\%) (Supplementary Table S3). The difference of HPV52 prevalence was also documented in women from PEC, ART, and GC, which is 23.9, 27.5, and $15.2 \%$, respectively.

\section{Factors Associated With C. trachomatis and HPV Infection}

The variables associated with $C$. trachomatis infection statistically significant were age $(P<0.01)$ and HPV infection $(P<0.01$, Table 1 and Supplementary Table S4), which were further confirmed by the multivariate analysis (Table 2). However, C. trachomatis infection was not associated with single or multiple HPV infections $(P>0.05)$, or the genotypes of HPV $(P>0.05$, Supplementary Table S4).

As shown in Table 2, HPV infection was independently associated with age $(P<0.01)$ and $C$. trachomatis infection $(P<0.01$, Table 2). However, there was no correlation between $C$. trachomatis and HPV genotype distribution among participants with successful genotyping of both pathogens $(P>0.05)$ (Supplementary Table S7). 
TABLE 1 | Prevalence of C. trachomatis and HPV infection of women in Southern Hunan province in China.

\begin{tabular}{|c|c|c|c|c|c|}
\hline \multirow[t]{2}{*}{ Characteristic } & \multirow[t]{2}{*}{ Sample size $(n)$} & \multirow[t]{2}{*}{ Age, $y$ (Mean \pm SD) } & \multicolumn{3}{|c|}{ Prevalence [n (\%)] } \\
\hline & & & $\mathrm{CT}^{+}$ & HPV $^{+}$ & $\mathrm{CT}^{+} / \mathrm{HPV}^{+}$ \\
\hline Total & 5006 & $36.4 \pm 10.7$ & $236(4.7)$ & $778(15.5)$ & $59(1.2)$ \\
\hline \multicolumn{6}{|l|}{ Clinical departments } \\
\hline PEC & 1006 & $39.4 \pm 11.1$ & $38(3.8)$ & $109(10.8)$ & $6(0.6)$ \\
\hline ART & 666 & $33.4 \pm 5.5$ & $23(3.5)$ & $69(10.4)$ & $5(0.8)$ \\
\hline Gynecology Outpatient & 3334 & $36.1 \pm 11.1$ & $175(5.2)$ & $600(18.0)$ & $48(1.4)$ \\
\hline$P$ & & & 0.043 & $<0.001$ & 0.053 \\
\hline \multicolumn{6}{|l|}{ Age (years) } \\
\hline$\leq 25$ & 567 & NA & $59(10.4)$ & $121(21.3)$ & $22(3.8)$ \\
\hline$>25$ & 4439 & NA & $177(4.0)$ & $656(14.8)$ & $37(0.8)$ \\
\hline 26-35 & 2122 & NA & $104(4.9)$ & $321(15.1)$ & $21(1.0)$ \\
\hline $36-45$ & 1222 & NA & $45(3.7)$ & $147(12.0)$ & $7(0.6)$ \\
\hline$\geq 46$ & 1095 & NA & $28(2.6)$ & $188(17.2)$ & $9(0.8)$ \\
\hline$P$ & & & $<0.001$ & $<0.001$ & $<0.001$ \\
\hline
\end{tabular}

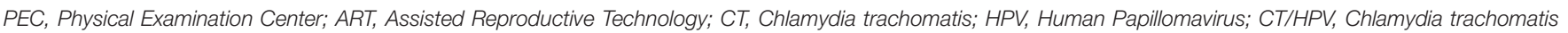
and Human Papillomavirus co-infection; NA, Not Applicable.

TABLE 2 | Factors associated with Chlamydia trachomatis or HPV infection by multivariate analysis.

\begin{tabular}{|c|c|c|c|c|c|}
\hline $\begin{array}{l}\text { Characteristic } \\
\text { Ages, y }\end{array}$ & Odds Ratio (95\% Confidence Interval) & $P^{*}$ & $\begin{array}{c}\text { Characteristic } \\
\text { Ages, y }\end{array}$ & Odds Ratio (95\% Confidence Interval) & $P^{*}$ \\
\hline$\leq 25$ & 1 (Reference) & $<0.001$ & $\leq 25$ & 1 (Reference) & $<0.001$ \\
\hline $26-35$ & 0.496(0.354-0.697) & & $26-35$ & $0.721(0.571-0.910)$ & \\
\hline $36-45$ & $0.379(0.252-0.569)$ & & $36-45$ & $0.557(0.427-0.726)$ & \\
\hline$\geq 46$ & $0.240(0.151-0.383)$ & & $\geq 46$ & $0.823(0.637-1.064)$ & \\
\hline HPV infection & & & CT infection & & \\
\hline $\mathrm{HPV}^{-}$ & 1 (Reference) & $<0.001$ & $\mathrm{CT}^{-}$ & 1 (Reference) & $<0.001$ \\
\hline $\mathrm{HPV}^{+}$ & $1.710(1.254-2.332)$ & & $\mathrm{CT}^{+}$ & 1.716(1.259-2.339) & \\
\hline
\end{tabular}

*By Hosmer-Lemeshow Satterthwaite adjusted F-test. CT, Chlamydia trachomatis; HPV, Human Papillomavirus.

\section{Associations of Colposcopy Impression Features With C. trachomatis and HPV Infection}

To investigate the association between cervical abnormalities and C. trachomatis/HPV infection, colposcopy data were collected from 151 outpatients from GC. Cervical abnormal features were found in $37.7 \%$ (57/151) of the patients while low and high grade features, as well as cervical cancer, accounted for $75.4 \%(43 / 57), 17.5 \%(10 / 57)$, and $7.0 \%$ (4/57), respectively (Table 5). For HPV-infected outpatients, the prevalence of low and high grade features as well as cervical cancer was $42.6 \%(29 / 68), 11.8 \%(8 / 68)$, and $5.9 \%$ (4/68), respectively (Table 5). Of note, out of 4 cancer patients further confirmed by pathological reports (data not shown), 3 were infected with HrHPV and 1 with LrHPV. Only low and high grade features were observed in $38.2 \%$ $(13 / 34)$ and $5.9 \%(2 / 34)$ of C. trachomatis-infected patients, respectively (Table 5).

Furthermore, HPV infection was strongly associated with an abnormal colposcopy impression of both low-grade (OR: 6.872, 95\% CI: 2.894-16.317, $P<0.001$ ) and high-grade (OR: 15.863, 95\% CI: 3.144-80.03, $P<0.001$, Supplementary Table S8).
However, our study showed that CT infection was only associated with low-grade colposcopy abnormality with OR of 3.248 (95\% CI: $1.22-8.646, P=0.018)$.

TABLE 3 | Genotype distribution of 229 urogenital Chlamydia trachomatis stains by clinical departments.

\begin{tabular}{|c|c|c|c|c|c|}
\hline \multirow{2}{*}{$\begin{array}{l}\text { CT } \\
\text { genotypes }\end{array}$} & \multicolumn{4}{|c|}{ Clinical departments [n (\%)] } & \multirow[t]{2}{*}{$P$} \\
\hline & PEC & ART & $\begin{array}{l}\text { Gynecology } \\
\text { Outpatients }\end{array}$ & Total & \\
\hline $\mathrm{E}$ & $8(21.6)$ & $7(31.8)$ & $54(31.8)$ & $69(30.1)$ & 0.78 \\
\hline $\mathrm{F}$ & $12(32.4)$ & $3(13.6)$ & $38(22.4)$ & $53(23.1)$ & \\
\hline J & $6(16.2)$ & $5(22.7)$ & $30(17.6)$ & $41(17.9)$ & \\
\hline D & $4(10.8)$ & $4(18.2)$ & $23(13.5)$ & $31(13.5)$ & \\
\hline G & $3(8.1)$ & $1(4.5)$ & $10(5.9)$ & $14(6.1)$ & \\
\hline $\mathrm{H}$ & $3(8.1)$ & $2(9.1)$ & $6(3.5)$ & $11(4.8)$ & \\
\hline K & $1(2.7)$ & $0(0)$ & $8(4.7)$ & $9(3.9)$ & \\
\hline B & $0(0)$ & $0(0)$ & $1(0.6)$ & $1(0.4)$ & \\
\hline Total & 37 (100) & 22 (100) & 170 (100) & 229 (100) & \\
\hline
\end{tabular}

PEC, Physical Examination Center; ART: Assisted Reproductive Technology; CT: Chlamydia trachomatis. 


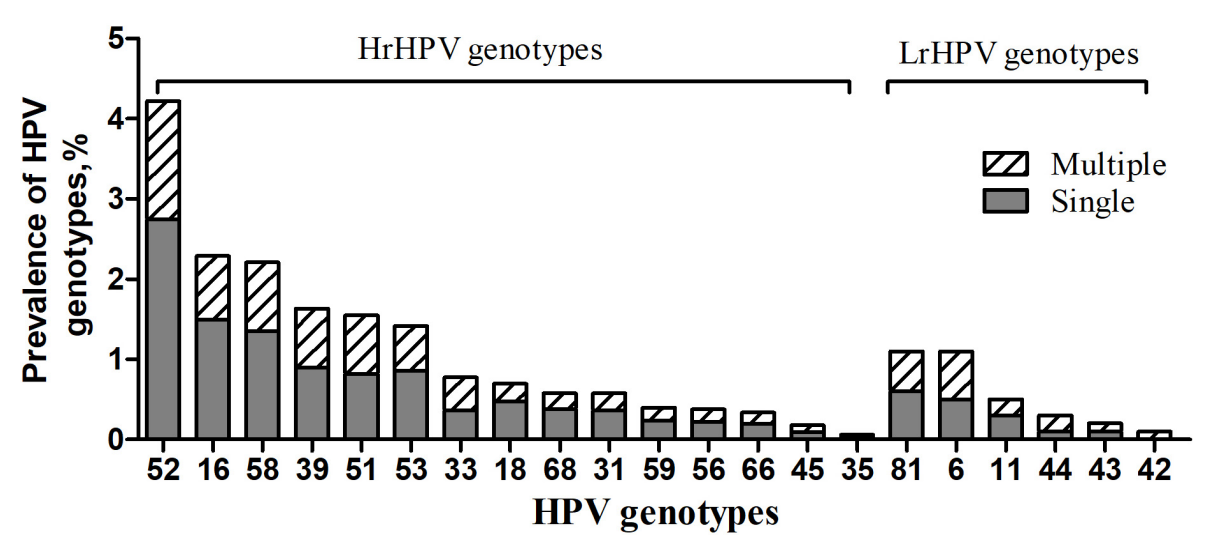

FIGURE 1 | Prevalence of HrHPV and LrHPV genotypes in single and multiple HPV infections. The most common HrHPV genotype detected was HPV 52(4.2\%), followed by HPV 16(2.3\%), HPV 58(2.2\%), HPV 39(1.6\%), HPV 51(1.6\%), HPV 53(1.4\%), HPV 33(0.8\%), HPV 18(0.7\%), HPV 68(0.6\%), HPV 31(0.6\%), HPV 59(0.4\%), HPV 56(0.4\%), HPV 66(0.3\%), HPV 45(0.2\%) and HPV 35(0.1\%). For the LrHPV genotypes, the most common type was HPV 83(1.1\%), followed by HPV 6(1.0\%), HPV 11(0.4\%), HPV 44(0.3\%), HPV 43(0.2\%) and HPV 42(0.1\%). HrHPV: High-risk HPV; LrHPV: Low-risk HPV.

TABLE 4 | Prevalence of low-risk and high-risk of HPV genotypes in 5023 women by C. trachomatis positivity, clinical departments and ages.

\begin{tabular}{|c|c|c|c|c|c|}
\hline \multirow[t]{2}{*}{ Characteristic } & \multirow[t]{2}{*}{ Sample Size (n) } & \multicolumn{3}{|c|}{ HPV genotypes [n (\%)] } & \multirow[t]{2}{*}{$\boldsymbol{P}$} \\
\hline & & LrHPV only & HrHPV only & Mixed Hr/LrHPV & \\
\hline Total & 5006 & $77(1.5)$ & $648(12.9)$ & $53(1.1)$ & \\
\hline C. trachomatis infection & & & & & 0.24 \\
\hline $\mathrm{CT}^{-}$ & 4770 & $71(1.5)$ & $596(12.5$ & $52(1.1)$ & \\
\hline $\mathrm{CT}^{+}$ & 236 & $6(2.5)$ & $52(22.0)$ & $1(0.4)$ & \\
\hline$P$ & & 0.509 & 0.01 & 0.45 & \\
\hline \multicolumn{6}{|l|}{ Clinical departments } \\
\hline PEC & 1006 & $9(0.9)$ & $98(9.7)$ & $2(0.2)$ & 0.45 \\
\hline ART & 666 & $7(1.1)$ & $60(9.7)$ & $2(0.3)$ & \\
\hline Gynecology Outpatient & 3343 & $61(1.8)$ & $490(14.6)$ & $49(1.5)$ & \\
\hline$P$ & & 0.018 & $<0.001$ & $<0.001$ & \\
\hline \multicolumn{6}{|l|}{ Age, y } \\
\hline$\leq 25$ & 567 & $16(2.8)$ & $92(16.2)$ & 13(2.3) & 0.61 \\
\hline $26-35$ & 2122 & $31(1.5)$ & $274(12.9)$ & $17(0.8)$ & \\
\hline $36-45$ & 1222 & $12(1.0)$ & $125(10.2)$ & $10(0.8)$ & \\
\hline$\geq 46$ & 1095 & $18(1.6)$ & $157(14.3)$ & $13(1.2)$ & \\
\hline$P$ & & $<0.001$ & 0.012 & 0.011 & \\
\hline
\end{tabular}

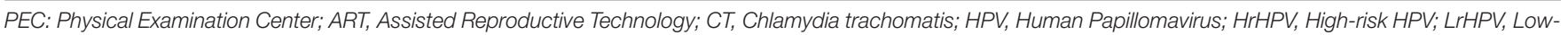
risk HPV.

\section{DISCUSSION}

The prevalence and genotype distribution of C. trachomatis and HPV in women differ considerably between nations and regions even within a country. $\mathrm{Li}$ and colleagues have reported that the prevalence of C. trachomatis and HPV, as well as C. trachomatis/HPV coinfection was $14.3,36.0$, and $4.8 \%$, respectively in Northern part of Inner Mongolia, China (Ji et al., 2019). Nearly $2.2 \%$ of married women were found to be infected with C. trachomatis, and $7.0 \%$ of them were infected with high risk HPV in Beijing, China (Zhang et al., 2017). In our study, we found that the prevalence of $C$. trachomatis, HPV, and C. trachomatis/HPV coinfection was $4.7,15.5$, and $1.2 \%$, respectively in the Southern part of Hunan, China. To our best knowledge, this is the first large observational investigation into C. trachomatis and HPV infection in women of Southern China. Our results support the prevalence of $C$. trachomatis and HPV among various areas in China and highlight the importance of epidemiological studies in different regions for the big area and huge population size.

Both C. trachomatis and HPV are characterized by symptomless infection. We found that the prevalence of asymptomatic C. trachomatis, $\mathrm{HPV}$, and C. trachomatis/HPV co-infection was $3.8 \%$ (38/1006), 10.8\% (109/1006), and 0.6\% (6/1006), respectively in women attending annual physical examination who are believed to be healthy general females, 
TABLE 5 | Cytology-associated C. trachomatis and HPV infection among 151 Cases.

\begin{tabular}{|c|c|c|c|c|c|}
\hline & \multirow{2}{*}{$\begin{array}{l}\text { Sample } \\
\text { Size }(n)\end{array}$} & \multicolumn{3}{|c|}{ Colposcopy impression [n (\%)] } & \multirow[t]{2}{*}{$\boldsymbol{P}$} \\
\hline & & Benign & Low grade & High grade & \\
\hline $\begin{array}{l}\text { CT and HPV } \\
\text { infection }\end{array}$ & 151 & 94 & 43 & 14 & \\
\hline $\mathrm{CT}^{+} / \mathrm{HPV}^{+}$ & 9 & $3(33.3)$ & $6(66.7)$ & 0 & $<0.001$ \\
\hline $\mathrm{CT}^{-} / \mathrm{HPV}^{-}$ & 58 & $51(87.9)$ & $7(12.1)$ & 0 & \\
\hline $\mathrm{CT}^{+} / \mathrm{HPV}^{-}$ & 25 & $16(64.0)$ & $7(28.0)$ & $2(8.0)$ & \\
\hline $\mathrm{CT}^{-} / \mathrm{HPV}^{+}$ & 59 & $24(40.7)$ & $23(39.0)$ & $12(20.3)$ & \\
\hline$P$ & & $<0.001$ & $<0.001$ & $<0.001$ & \\
\hline HPV infection & 151 & 94 & 43 & 14 & \\
\hline LrHPV & 8 & $3(37.5)$ & $4(50.0)$ & $1(12.5)$ & $<0.001$ \\
\hline HrHPV & 53 & $23(43.4)$ & $23(43.4)$ & 7 (13.2) & \\
\hline $\begin{array}{l}\text { Mixed HrHPV } \\
\text { and LrHPV }\end{array}$ & 7 & $1(14.3)$ & 2 (28.6) & $4(57.1)$ & \\
\hline Negative & 83 & 67 (80.7) & 14 (16.9) & $2(2.4)$ & \\
\hline$P$ & & $<0.001$ & 0.044 & $<0.001$ & \\
\hline
\end{tabular}

CT, Chlamydia trachomatis; HPV, Human Papillomavirus.

although a slightly higher prevalence of C. trachomatis and HPV infection was documented in outpatients from GC. These findings highlighted the importance of asymptomatic infection of C. trachomatis and HPV infection in the general population, particularly in young women of less than 25 years. These results are consistent with previous findings and further support that young women should be the target population for the prevention of C. trachomatis and HPV (Samoff et al., 2005; Bellaminutti et al., 2014). Importantly, women from ART had a relatively high prevalence of $C$. trachomatis (3.5\%) and HPV (10.4\%) infection, especially among women with secondary infertility (Shi et al., 2001), indicating the necessary C. trachomatis and HPV screening for women of childbearing age.

In our study, only $1.2 \%$ of the participants were coinfected with C. trachomatis and HPV. Furthermore, 25.0\% of C. trachomatis infection and $7.6 \%$ of HPV infection were attributable to C. trachomatis and HPV coinfection. Multivariate analysis also indicated that $C$. trachomatis or HPV infection increased the risk of HPV or C. trachomatis co-infection. These results were further confirmed by a case-control study in which the potential confounding factors of age and sources of the participants were matched. Besides, we also found both C. trachomatis and HPV infection were associated with colposcopy impression. These results suggest that C. trachomatis and HPV may act as mutual risk factors for their infection, and increase the risk of cervical lesions (Naldini et al., 2019). An alternative explanation for the tight association of C. trachomatis and HPV infection could also be their shared infection routes (Ji et al., 2019).

Interestingly, we found that the overall HPV-positive rate was $15.5 \%$, which was relatively lower than our previous estimated prevalence of $30.16 \%$ in 2009 and that of $16.8-36 \%$ reported in other studies in China (Li et al., 2006; Ji et al., 2019). The decreasing prevalence of HPV infection was probably due to expanding sexual safety education, and continuous improvements of living conditions and health in the past decade in China, although the sensitivities of testing assays may be different among the studies. Notably, $14 \%$ of the participants were positive for HrHPV while only $2.6 \%$ were positive for LrHPV, indicating the seriousness of high-risk genotypes of HPV infection and cervical cancer. Of note, our results were lower than the average HrHPV infection rate of $19 \%$ reported by $\mathrm{Li}$ and colleagues ( $\mathrm{Li}$ et al., 2019).

Several studies have described HPV-16 as the most prevalent genotype in China (Bellaminutti et al., 2014). However, the genotype distribution of HPV in Southern Chinese women appears to be different (Ji et al., 2019). We found that HPV52 (4.2\%), HPV16 (2.3\%) and HPV58 (2.2\%) were the three dominant HrHPV genotypes in women. These results are in line with the data from other Chinese populations in which both HPV52 and 58 are the predominant type among the general population in China (Bi et al., 2015; Wang et al., 2015). In Eastern Asia, infection of HP52 and 58 also resulted in 2.5-2.8- and 3.74.9-fold higher risk for cervical intraepithelial neoplasia (CIN) and invasive cancer than in other regions (Chan et al., 2014). The HPV vaccine is an important strategy for the prevention of cervical cancer and has been implemented for more than 10 years. However, HPV vaccination was launched until July 2017 in mainland China. Moreover, the first domestic bivalent HPV vaccine was licensed by the China Food and Drug Administration (CFDA) on Jan, 2020. Currently, very few Chinese women have been vaccinated due to a variety of reasons including inaccessible vaccine, economic, educational, and so on. Our results indicate that future HPV vaccine should cover HPV genotype 52, 58, and 16 when used in Southern part of China.

We found that serotype E (30.1\%), F (23.1\%) and J (17.9\%) were the top three $C$. trachomatis serovars in the current study. Similar results were found in other parts of China (Yang et al., 2010), with serotype E being predominant in the South (32\%) and East $(27 \%)$, and $\mathrm{F}$ in the Southwest (28\%) in China (Hsu et al., 2006; Gao et al., 2007). While in Thailand, serotype F (35\%) and $\mathrm{E}(18 \%)$ was the most prevalent genotypes whereas $\mathrm{F}(32 \%)$ and $\mathrm{E}(17 \%)$ dominated in Japan, and F (31\%) and E (23\%) in Netherlands (Yamazaki et al., 2005).

We noted no significant associations between C. trachomatis and HPV genotype distribution by using correlation analysis, and also found no particular associations of Lr- and HrHPV genotypes, and single and multiple HPV infections with C. trachomatis infection in the general population, indicating that type-specific deviations from the overall association between C. trachomatis and HPV acquisition are small. Nevertheless, an association between $C$. trachomatis and HrHPV genotypes in female adolescents has been demonstrated previously (Samoff et al., 2005). Again, this discrepancy could be possibly explained by the geographic and within-population variation, and the small sample size of women with coinfections in the current study.

It is worth mentioning that $C$. trachomatis serovars $\mathrm{E}, \mathrm{D}$, and $J$ account for $70 \%$, and HPV 52, 51 and 39 for $36 \%$ of coinfection cases. This distribution of HPV genotypes contrasts with the report that HPV16 was the most represented in women without C. trachomatis coinfection (Seraceni et al., 2016), but 
in agreement with the recent Italian data that described these genotypes as the most frequent types associated with coinfections (Carozzi et al., 2014) in which HPV 52, 51, and 39 of groups $1,2 \mathrm{~A}$ or $2 \mathrm{~B}$ have been classified as cervical carcinogens (Clarke et al., 2018). More interestingly, HPV 52 was also the genotype that is most frequently detected among women from ART, which reinforced the notion that HPV 52 was most commonly found in couples undergoing in vitro fertilization treatment, and in the case of low sperm concentration and reduced sperm motility, as recently presented from a Lithuanian report (Jersoviene et al., 2019).

Some limits, however, existed in this study: (1) the participants were from a hospital, not from a community population. Thus, the results might not reflect the infection of $C$. trachomatis and HPV in the general population; (2) lack of detailed information on the history of women with $C$. trachomatis or HPV infection and histological changes prevented the identification of the risk factors for C. trachomatis and HPV infection; and (3) nucleic acid detection of $C$. trachomatis and HPV by PCR only reflects current infection. The prevalence of C. trachomatis and HPV may be underestimated (Moscicki et al., 1998).

In conclusion, a large observational study was conducted to investigate the prevalence and genotype distribution of both C. trachomatis and HPV in women in the Southern part of China. Overall, the prevalence of C. trachomatis and HPV infection was similar in healthy women attending physical examination or seeking reproductive assistance to the outpatients GC, indicating the importance of asymptomatic C. trachomatis and HPV infection, particularly among females aged less than 25 years. C. trachomatis or HPV infection could increase the risk of HPV or C. trachomatis co-infection. These observations highlight the need for routine $C$. trachomatis and HPV screening and monitoring.

\section{DATA AVAILABILITY STATEMENT}

The raw data supporting the conclusions of this article will be made available by the authors, without undue reservation, to any qualified researcher.

\section{REFERENCES}

Bellaminutti, S., Seraceni, S., De Seta, F., Gheit, T., Tommasino, M., and Comar, M. (2014). HPV and Chlamydia trachomatis co-detection in young asymptomatic women from high incidence area for cervical cancer. J. Med. Virol. 86, 19201925. doi: 10.1002/jmv.24041

Bhatla, N., Puri, K., Joseph, E., Kriplani, A., Iyer, V. K., and Sreenivas, V. (2013). Association of Chlamydia trachomatis infection with human papillomavirus (HPV) \& cervical intraepithelial neoplasia - a pilot study. Indian J. Med. Res. 137, 533-539.

Bi, Q., Zhang, L., Zhao, Z., Mu, X., Zhang, M., and Wang, P. (2015). Human papillomavirus prevalence and genotypes distribution among female outpatients in Qingdao, East China. J. Med. Virol. 87, 2114-2121. doi: 10.1002/ jmv. 24281

Carozzi, F., De Marco, L., Gillio-Tos, A., Del Mistro, iA., Girlando, S., Baboci, L., et al. (2014). Age and geographic variability of human papillomavirus high-risk genotype distribution in a large unvaccinated population and of vaccination impact on HPV prevalence. J. Clin. Virol. 60, 257-263. doi: 10.1016/j.jcv.2014. 04.009

\section{ETHICS STATEMENT}

The studies involving human participants were reviewed and approved by the Ethics Committee of Chenzhou No. 1 People's Hospital. The patients/participants provided their written informed consent to participate in this study.

\section{AUTHOR CONTRIBUTIONS}

ZL, ST, and HC designed the study. HC, LL, YW, BH, and $\mathrm{HL}$ contributed to $\mathrm{CT}$ and HPV detection. $\mathrm{XH}$ and $\mathrm{PH}$ did the colposcopy test. ZL, XH, PH, and LL helped with data management. HC and JS contributed to statistical analysis. HL and ST drafted the manuscript. All authors commented on the draft and decision to submit.

\section{FUNDING}

This work was supported by NSFC (81802022 and 81772210), Hunan Science and Technology innovation project (2018SK50309), Hunan Health Commission project (B2019002) and Chenzhou Science and technology Bureau (yfzx201908).

\section{ACKNOWLEDGMENTS}

We thank Dr. Jian Zhang (Chenzhou No. 1 People's Hospital) for providing professional assistance to statistics and Dr. Chenxu Guo (The Johns Hopkins University, School of Medicine) for the careful reading of the manuscript.

\section{SUPPLEMENTARY MATERIAL}

The Supplementary Material for this article can be found online at: https://www.frontiersin.org/articles/10.3389/fmicb. 2020.00827/full\#supplementary-material

Chan, P. K., Ho, W. C., Chan, M. C., Wong, M. C., Yeung, A. C., Chor, J. S., et al. (2014). Meta-analysis on prevalence and attribution of human papillomavirus types 52 and 58 in cervical neoplasia worldwide. PLoS One 9:e107573. doi: 10.1371/journal.pone.0107573

Chen, H., Wen, Y., and Li, Z. (2019). Clear victory for chlamydia: the subversion of host innate immunity. Front. Microbiol. 10:1412. doi: 10.3389/fmicb.2019. 01412

Clarke, M. A., Gradissimo, A., Schiffman, M., Lam, J., Sollecito, C. C., Fetterman, B., et al. (2018). Human papillomavirus DNA methylation as a biomarker for cervical precancer: consistency across 12 genotypes and potential impact on management of HPV-positive women. Clin. Cancer Res. 24, 2194-2202. doi: 10.1158/1078-0432.CCR-17-3251

Conde-Ferraez, L., Martiez, J. R., Ayora-Talavera, G., and Losa, M. D. (2017). Human papillomavirus and Chlamydia trachomatis infection in gynecoobstetric outpatients from a mexican hospital. Indian J. Med. Microbiol. 35, 74-79. doi: 10.4103/ijmm.IJMM_15_450

Di Pietro, M., Filardo, S., Porpora, M. G., Recine, N., Latino, M. A., and Sessa, R. (2018). HPV/Chlamydia trachomatis co-infection: metagenomic analysis of cervical microbiota in asymptomatic women. New Microbiol. 41, 34-41. 
Erickson, B. K., Alvarez, R. D., and Huh, W. K. (2013). Human papillomavirus: what every provider should know. Am. J. Obstet. Gynecol. 208, 169-175. doi: 10.1016/j.ajog.2012.09.007

Gao, X., Chen, X. S., Yin, Y. P., Zhong, M. Y., Shi, M. Q., Wei, W. H., et al. (2007). Distribution study of Chlamydia trachomatis serovars among high-risk women in China performed using PCR-restriction fragment length polymorphism genotyping. J. Clin. Microbiol. 45, 1185-1189. doi: 10.1128/JCM.02076-06

Geisler, W. M., Suchland, R. J., Whittington, W. L., and Stamm, W. E. (2003). The relationship of serovar to clinical manifestations of urogenital Chlamydia trachomatis infection. Sex Transm. Dis. 30, 160-165. doi: 10.1097/00007435200302000-00013

Griffais, R., and Thibon, M. (1989). Detection of Chlamydia trachomatis by the polymerase chain reaction. Res. Microbiol. 140, 139-141. doi: 10.1016/09232508(89)90047-8

Hadfield, J., Benard, A., Domman, D., and Thomson, N. (2018). The hidden genomics of Chlamydia trachomatis. Curr. Top. Microbiol. Immunol. 412, 107-131. doi: 10.1007/82_2017_39

Harden, M. E., and Munger, K. (2017). Human papillomavirus molecular biology. Mutat. Res. Rev. Mutat. Res. 772, 3-12. doi: 10.1016/j.mrrev.2016.07.002

Hsu, M. C., Tsai, P. Y., Chen, K. T., Li, L. H., Chiang, C. C., Tsai, J. J., et al. (2006). Genotyping of Chlamydia trachomatis from clinical specimens in Taiwan. J. Med. Microbiol. 55, 301-308. doi: 10.1099/jmm.0.46262-0

Jersoviene, V., Gudleviciene, Z., Rimiene, J., and Butkauskas, D. (2019). Human papillomavirus and infertility. Medicina (Kaunas) 55:E377. doi: 10.3390/ medicina55070377

Ji, Y., Ma, X. X., Li, Z., Peppelenbosch, M. P., Ma, Z., and Pan, Q. (2019). The burden of human papillomavirus and chlamydia trachomatis coinfection in women: a large cohort study in inner mongolia. China J. Infect. Dis. 219, 206-214. doi: 10.1093/infdis/jiy497

Khan, M. J., Werner, C. L., Darragh, T. M., Guido, R. S., Mathews, C., Moscicki, A. B., et al. (2017). ASCCP colposcopy standards: role of colposcopy, benefits, potential harms, and terminology for colposcopic practice. J. Low Genit. Tract. Dis. 21, 223-229. doi: 10.1097/LGT.0000000000000338

Li, K., Li, Q., Song, L., Wang, D., and Yin, R. (2019). The distribution and prevalence of human papillomavirus in women in mainland China. Cancer 125, 1030-1037. doi: 10.1002/cncr.32003

Li, L. K., Dai, M., Clifford, G. M., Yao, W. Q., Arslan, A., Li, N., et al. (2006). Human papillomavirus infection in Shenyang City, People's Republic of China: a population-based study. Br. J. Cancer 95, 1593-1597. doi: 10.1038/sj.bjc. 6603450

Moscicki, A. B., Shiboski, S., Broering, J., Powell, K., Clayton, L., Jay, N., et al. (1998). The natural history of human papillomavirus infection as measured by repeated DNA testing in adolescent and young women. J. Pediatr. 132, 277-284. doi: 10.1016/s0022-3476(98)70445-7

Naldini, G., Grisci, C., Chiavarini, M., and Fabiani, R. (2019). Association between human papillomavirus and chlamydia trachomatis infection risk in women: a systematic review and meta-analysis. Int. J. Public Health 64, 943-955. doi: 10.1007/s00038-019-01261-w

Parish, W. L., Laumann, E. O., Cohen, M. S., Pan, S., Zheng, H., Hoffman, I., et al. (2003). Population-based study of chlamydial infection in China: a hidden epidemic. JAMA 289, 1265-1273. doi: 10.1001/jama.289.10. 1265
Rawre, J., Juyal, D., and Dhawan, B. (2017). Molecular typing of Chlamydia trachomatis: an overview. Indian J. Med. Microbiol. 35, 17-26. doi: 10.4103/ ijmm.IJMM_16_341

Samoff, E., Koumans, E. H., Markowitz, L. E., Sternberg, M., Sawyer, M. K., Swan, D., et al. (2005). Association of Chlamydia trachomatis with persistence of highrisk types of human papillomavirus in a cohort of female adolescents. Am. J. Epidemiol. 162, 668-675. doi: 10.1093/aje/kwi262

Schiffman, M., Doorbar, J., Wentzensen, N., de Sanjose, S., Fakhry, C., Monk, B. J., et al. (2016). Carcinogenic human papillomavirus infection. Nat. Rev. Dis. Primers. 2:16086. doi: 10.1038/nrdp.2016.86

Seraceni, S., Campisciano, G., Contini, C., and Comar, M. (2016). HPV genotypes distribution in Chlamydia trachomatis co-infection in a large cohort of women from north-east Italy. J. Med. Microbiol. 65, 406-413. doi: 10.1099/jmm.0. 000245

Shi, X. B., Liu, F. Y., and Zhang, H. W. (2001). Study of Chlamydia trachomatis infection on cervical secretion of women with early pregnancy and secondary sterility. Hunan Yi Ke Da Xue Xue Bao 26, 169-170.

Wang, R., Guo, X. L., Wisman, G. B., Schuuring, E., Wang, W. F., Zeng, Z. Y., et al. (2015). Nationwide prevalence of human papillomavirus infection and viral genotype distribution in 37 cities in China. BMC Infect. Dis. 15:257. doi: 10.1186/s12879-015-0998-5

Wiesenfeld, H. C., Sweet, R. L., Ness, R. B., Krohn, M. A., Amortegui, A. J., and Hillier, S. L. (2005). Comparison of acute and subclinical pelvic inflammatory disease. Sex Transm. Dis. 32, 400-405. doi: 10.1097/01.olq.0000154508. 26532.6a

Witkin, S. S., Minis, E., Athanasiou, A., Leizer, J., and Linhares, I. M. (2017). Chlamydia trachomatis: the persistent pathogen. Clin. Vaccine Immunol. 24, e203-e217. doi: 10.1128/CVI.00203-17

Yamazaki, T., Hagiwara, T., Kishimoto, T., Sasaki, N., Takahashi, S., Ishihara, O., et al. (2005). Distribution of Chlamydia trachomatis serovars among female prostitutes and non-prostitutes in Thailand, and non-prostitutes in Japan during the mid-90s. Jpn. J. Infect. Dis. 58, 211-213.

Yang, B., Zheng, H. P., Feng, Z. Q., Xue, Y. H., Wu, X. Z., Huang, J. M., et al. (2010). The prevalence and distribution of Chlamydia trachomatis genotypes among sexually transmitted disease clinic patients in Guangzhou, China, 2005-2008. Jpn. J. Infect. Dis. 63, 342-345.

Zhang, D., Li, T., Chen, L., Zhang, X., Zhao, G., and Liu, Z. (2017). Epidemiological investigation of the relationship between common lower genital tract infections and high-risk human papillomavirus infections among women in Beijing. China. PLoS One 12, e0178033. doi: 10.1371/journal.pone.0178033

Conflict of Interest: The authors declare that the research was conducted in the absence of any commercial or financial relationships that could be construed as a potential conflict of interest.

Copyright (c) 2020 Chen, Luo, Wen, He, Ling, Shui, He, Hou, Tang and Li. This is an open-access article distributed under the terms of the Creative Commons Attribution License (CC BY). The use, distribution or reproduction in other forums is permitted, provided the original author(s) and the copyright owner(s) are credited and that the original publication in this journal is cited, in accordance with accepted academic practice. No use, distribution or reproduction is permitted which does not comply with these terms. 